\title{
Physiologically Based Construction of Optimized 3-D Arterial Tree Models ${ }^{\star}$
}

\author{
Matthias Schneider ${ }^{1}$, Sven Hirsch ${ }^{1}$, Bruno Weber $^{2}$, and Gábor Székely ${ }^{1}$ \\ ${ }^{1}$ Computer Vision Laboratory, ETH Zurich, Switzerland \\ ${ }^{2}$ Institute of Pharmacology and Toxicology, University of Zurich, Zurich, Switzerland
}

\begin{abstract}
We present an approach to generate 3-D arterial tree models based on physiological principles while at the same time certain morphological properties are enforced at construction time in order to build individual vascular models down to the capillary level. The driving force of our approach is an angiogenesis model incorporating case-specific information about the metabolic activity in the considered domain. Additionally, we enforce morphometrically confirmed bifurcation statistics of vascular networks. The proposed method is able to generate artificial, yet physiologically plausible, arterial tree models that match the metabolic demand of the embedding tissue and fulfill the enforced morphological properties at the same time. We demonstrate the plausibility of our method on synthetic data for different metabolic configurations and analyze physiological and morphological properties of the generated tree models.
\end{abstract}

Keywords: vascular tree construction, arterial tree model, angiogenesis, morphological statistics, computer simulation, computational physiology.

\section{Introduction}

An in-depth understanding of the microvascular structure is required in many research areas both in normal and pathological tissue. As an example, detailed, explicit vascular models are needed in surgical training simulation, when generating variable anatomical scenes with realistic physiological properties [14]. Similarly, knowledge of the entire cerebrovascular network down to capillary level is required for gaining insight into blood flow dynamics and its regulation by numerical simulations [10]. Additionally, it has been shown that many of the neurodegenerative diseases (e.g. Alzheimer's disease) have a prominent vascular component, and there is increasing evidence that reduced energy substrate and oxygen delivery is in part responsible for the severe symptoms of the disease. This underlines the need for a better knowledge of the vascular network's structure in normal and pathological tissue in order to increase our understanding of the pathophysiological mechanisms. Microvascular structures have been analyzed based on intravascular dye injections, staining of vessel components, or vascular corrosion casts using state of the art imaging modalities, e.g., all-optical histology, or synchrotron radiation based $\mathrm{x}$-ray tomographic microscopy. Since the segmentation and reconstruction of consistent arterial trees still remains a challenge, numerous methods for the generation of artificial vascular models have been proposed.

\footnotetext{
* Supplementary material for this article is available at http://www.vision.ee.ethz.ch/ReCoVa
} 
The main approaches are typically based on optimality or evolutionary principles. Nekka et al. [9] proposed a deterministic construction method for 2-D vascular structures incorporating a highly simplified angiogenesis model. Vascular formation is modeled as evolutionary process in response to angiogenic factors produced by ischemic tissue cells. As the formation process is purely based on the distribution of angiogenic factors without imposing any further restriction regarding the geometry of the evolving network, the results appear rather artificial and too regular. A more sophisticated angiogenesis-based simulation framework for the construction of vascular systems in arbitrary anatomies has been proposed by Szczerba et al. [13]. Even though the results show high similarity with real vasculatures, the underlying simplifications still do not allow to generate vascular morphologies in full accordance with experimental findings.

Based on experimental observations from real vessel networks, several optimality principles have long been hypothesized, e.g., minimal building material or minimal energy dissipation [8 15]. Klarbring et al. [6] apply strategies for topology optimization of electrical networks and load carrying trusses to fluid mechanics. The flow network topology is optimized w.r.t. the cross-section of the pipes in order to minimize the total pressure loss (dissipation) under a total volume constraint.

Schreiner and Buxbaum [11] proposed to use constrained constructive optimization (CCO) to iteratively construct a 2-D binary tree. The method of CCO (and its variations) is a stochastic construction process purely based on structural optimality principles under hemodynamic boundary conditions. In order to generate visually more realistic vascular trees in 3-D, Karch et al. [5] combined CCO with staged tissue growth leading to structural changes of the simulated tree models. However, the vascular networks have been truncated at the pre-arteriolar scale. Similarly, CCO has recently been combined with time-dependent constraints on a level set distance function to restrict the morphology of major (cerebral) arteries, in particular [1].

In contrast, we pursue a modeling approach based on physiological principles while enforcing certain morphological properties at construction time in order to build individual vascular models down to the capillary level. In our method, we use an angiogenesis model as the driving force of vascular tree formation while relying on the metabolic activity of the tissue in the considered domain as may be obtained from functional imaging, for instance. At the same time we incorporate morphometrically confirmed optimality hypotheses concerning the branching structure of vascular systems. This way, we iteratively construct an arterial tree model that meets the metabolic requirements of the embedding tissue on a gradually growing domain. As opposed to previously suggested approaches, we do not explicitly enforce hemodynamic constraints.

\section{Methods}

Our approach for the generation of artificial arterial trees is based on physiological principles related to (sprouting) angiogenesis in order to achieve physiologically plausible results. Angiogenesis describes the formation of new capillary blood vessels from a pre-existing vasculature in well-characterized stages. It plays a crucial role in different growth processes such as embryonic development, wound healing, or tumor growth [7]. In chemotactic response to angiogenic signals, also known as angiogenic growth factors, solid capillary sprouts develop from pre-existing vessels by means of endothelial 
cell (EC) proliferation and migration towards the source of the angiogenic stimulus. We will reduce the complex signaling cascade involved in angiogenesis to a single transmitter, namely the vascular endothelial growth factor (VEGF), which has widely been studied and proven to be a potent stimulator of physiological and pathological angiogenesis [3]. VEGF, secreted by, e.g., tumor or ischemic cells, diffuses into the surrounding tissue establishing a concentration gradient between the source and the vessels. In our method, these physiological principles are applied on a gradually growing simulation domain to drive the iterative construction of an arterial tree model.

Arterial Tree Model. In this work, we consider arterial trees rather than full-fledged vascular networks. According to morphometric analysis, bifurcations of vascular trees almost invariably branch into two distal branches [15]. Arterial trees can hence be considered as binary trees. Each vessel segment is represented by an edge which is modeled as rigid cylindrical tube with radius $r$ and length $l$ connecting two nodes. This gives rise to four different types of nodes, namely root, leaf, bifurcation $(-\propto)$, and inter nodes $(-a)$. The latter links two successive segments and allows to approximate the shape of a tortuous vessel branch. Note that this model does not allow for interconnections (anastomoses). In the following, we will distinguish between tree segments (single edge between any two connected nodes) and branches (path from a bifurcation or root node to the next distal bifurcation or leaf node).

The relation of the decreasing vessel radii from proximal to distal segments is governed by a bifurcation law (Murray's Law) relating the radius of the proximal parent branch $r_{p}$ to the radii $r_{l}, r_{r}$ of the left and right daughter branches [8]: $r_{p}^{\gamma}=r_{l}^{\gamma}+r_{r}^{\gamma}$, where $\gamma$ denotes the bifurcation exponent, with values reported ranging from $\gamma=2.0$ to $\gamma=3.0$ [2]11].

The bifurcation geometry is further constrained w.r.t. the bifurcation angles based on fluid dynamic considerations [2]:

$$
\cos \left(\phi_{l}\right)=\frac{r_{p}^{4}+r_{l}^{4}-r_{r}^{4}}{2 r_{p}^{2} r_{l}^{2}}, \quad \cos \left(\phi_{r}\right)=\frac{r_{p}^{4}+r_{r}^{4}-r_{l}^{4}}{2 r_{p}^{2} r_{r}^{2}},
$$

with $\phi_{l}, \phi_{r}$ denoting the bifurcation angle of the left and right daughter branch, respectively. Geometrically, this corresponds to the optimal position of the branching point $\boldsymbol{p}_{b}$ minimizing the total lumen volume for fixed boundary points:

$$
\hat{\boldsymbol{p}}_{b}=\underset{\boldsymbol{p}_{b}}{\operatorname{argmin}} \sum_{k \in\{p, l, r\}} r_{k}^{2}\left\|\boldsymbol{p}_{k}-\boldsymbol{p}_{b}\right\|_{2},
$$

where $\boldsymbol{p}_{p}, \boldsymbol{p}_{l}, \boldsymbol{p}_{r}$ are the boundary nodes of the parent, left, and right segment, respectively (see Figure 19).

Angiogenesis Model. We use a simplified angiogenesis model as the driving force for our simulations. The model considers the mutual interplay of arterial oxygen $\left(\mathrm{O}_{2}\right)$ supply and VEGF secreted by ischemic cells. Tissue is assumed to be homogeneous w.r.t. $O_{2}$ and VEGF transport with diffusivity $D_{1}$ and $D_{2}$, respectively. Assuming steady-state conditions, Fick's first law postulates [12]:

$$
D_{1} \nabla^{2} c_{1}=R_{1}\left(c_{1}\right), \quad R_{1}\left(c_{1}\right)=R_{1}^{0} \frac{c_{1}}{c_{1}+c_{1}^{h}},
$$


where $c_{1}$ represents the $O_{2}$ concentration. $R_{1}\left(c_{1}\right)$ denotes the $O_{2}$ consumption rate which is described by a Michaelis-Menten relationship defined by the $\mathrm{O}_{2}$ demand at saturation level $R_{1}^{0}$ (unlimited $O_{2}$ supply) and the half-saturation concentration $c_{1}^{h}$. Note that $R_{1}^{0}$ is subject to regional variations, in general.

VEGF secretion in tissue is dependent on the average oxygenation level. However, the exact relationship has not yet been experimentally measured. We model the oxygendependent VEGF secretion rate as follows [3]:

$$
R_{2}\left(c_{1}\right)=\left\{\begin{array}{ll}
6 R_{2}^{0} & , c_{1} \leq t_{1}^{\text {low }} \\
\left(1+5\left(\frac{t_{1}^{\text {high }}-c_{1}}{t_{1}^{\text {high }}-t_{1}^{\text {low }}}\right) R_{2}^{0}\right. & , t_{1}^{\text {low }}<c_{1}<t_{1}^{\text {high }} \\
R_{2}^{0} & , c_{1} \geq t_{1}^{\text {high }}
\end{array} .\right.
$$

The secreted VEGF isotropically diffuses and is subject to natural decay [7]:

$$
D_{2} \nabla^{2} c_{2}=\nu_{2} c_{2}-R_{2}\left(c_{1}\right),
$$

with diffusivity $D_{2}$ and decay rate $\nu_{2}$, while neglecting VEGF uptake by EC.

Vascular Growth. Vascular growth is modeled as a chemotactic process w.r.t. the VEGF concentration. In general, we distinguish between apical growth at leaf nodes (apices) and sprouting at inter nodes. A capillary may either elongate or bifurcate into two similar branches resulting mostly in symmetric bifurcations. This decision is made randomly with a sigmoidal bifurcation probability $p_{b}(a)=\operatorname{sig}\left(-\eta\left(1-a / A_{0}\right)\right)$, where $a=l / r$ denotes the aspect ratio of the proximal branch with length $l$ and radius $r$. The probability distribution $p_{b}(a)$ can be considered as global bifurcation length statistics with adjustable shape and normalization factor $\eta$ and $A_{0}$, respectively. In case of elongation at a leaf node, the radius of the new distal segment is adopted from the proximal segment. The growth direction $\boldsymbol{d}_{g}$ is estimated by $\boldsymbol{d}_{g}=\nabla c_{2}(\boldsymbol{x}) /\left\|\nabla c_{2}(\boldsymbol{x})\right\|_{2}+\lambda_{g} \boldsymbol{d}_{s}$, where $\boldsymbol{d}_{s}$ denotes the (normalized) direction of the proximal segment and $\lambda_{g}$ is a constant weighting factor. The distal segment length and the lengths and radii of the two new branches are drawn from normal distributions, while enforcing a minimum radius constraint $r \geq r_{\min }$. According to the principle of minimal building material, the parent and daughter segments at a bifurcation node are coplanar [2]. The branching angles are computed according to Equation (1). The two distal daughter branches are arranged such that the angle between the VEGF gradient and the growth direction of either of the two segments is minimized (see Figure 11a).

Besides apical growth at leaf nodes, vessels may also build sprouts at inter nodes producing both symmetric and asymmetric bifurcations. The sprouting probability is defined as $p_{s}=\min \left(p_{b}\left(l_{p} / r\right), p_{b}\left(l_{d} / r\right)\right)$, where $r, l_{p}$, and $l_{d}$ denote the radius and length of the proximal and distal branch meeting at the inter node. Similar to the bifurcation case, the length and radius of the new sprout are normally distributed. The VEGF gradient determines the sprout direction.

Both sprouting inter nodes and bifurcating leaf nodes spoil the bifurcation law, in general. Additional tree rebalancing is required to reinforce optimality. To this end, the segment radii have to be updated along the path from the site of growth up to the root node according to Murray's Law. Adjusting the radii, in turn, impairs the optimality of the branching angles. However, the bifurcations can be rebalanced by relocating the bifurcation node according to Equation (2). 
Table 1. Main simulation parameters

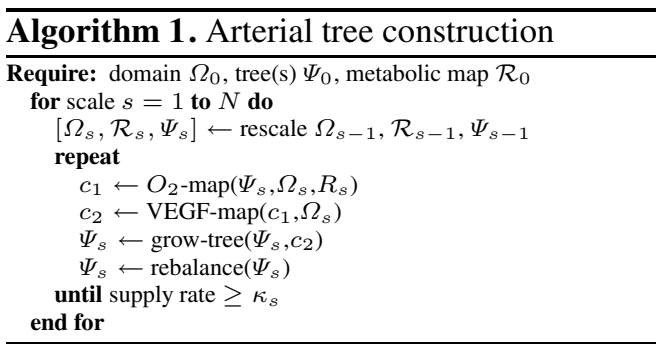

\begin{tabular}{l|c|c|c} 
Param & Value & Unit & Ref \\
\hline \hline$D_{1}$ & $2.41 \cdot 10^{-9}$ & $\mathrm{~m}^{2} \mathrm{~s}^{-1}$ & {$[4]$} \\
$R_{1}^{0}$ & $3.01 \cdot 10^{-3}$ & $\mathrm{~m}_{\mathrm{O}_{2}}^{3} \mathrm{~m}_{\text {tissue }}^{-3} \mathrm{~s}^{-1}$ & {$[\overline{4]}$} \\
$c_{1}^{h}$ & $1.95 \cdot 10^{-5}$ & $\mathrm{~m}_{\mathrm{O}_{2}}^{3} \mathrm{~m}_{\text {tissue }}^{-3}$ & {$[4]$} \\
\hline$D_{2}$ & $1.04 \cdot 10^{-10}$ & $\mathrm{~m}^{2} \mathrm{~s}^{-1}$ & {$[3]$} \\
$\nu_{2}$ & $1.81 \cdot 10^{-4}$ & $\mathrm{~s}^{-1}$ & {$[7]$} \\
$R_{2}^{0}$ & $2.50 \cdot 10^{-18}$ & $\mathrm{~mol} \mathrm{~m}^{-2} \mathrm{~s}^{-1}$ & {$[3]$} \\
$t_{1}^{\text {low }}$ & $3.89 \cdot 10^{-5}$ & $\mathrm{~m}_{\mathrm{O}_{2}}^{3} \mathrm{~m}_{\text {tissue }}^{-3}$ & {$[3]$} \\
$t_{1}^{\text {high }}$ & $7.78 \cdot 10^{-4}$ & $\mathrm{~m}_{\mathrm{O}_{2}}^{3} \mathrm{~m}_{\text {tissue }}^{-3}$ & {$[3$} \\
\hline$\gamma$ & 3 & $\mathrm{~B}_{1}$ & - \\
$\theta_{1}$ & $1.5 \cdot R_{1}^{0}$ & $\mathrm{~m}_{\mathrm{O}_{2}}^{3} \mathrm{~m}_{\text {tissue }}^{-3} \mathrm{~s}^{-1}$ & - \\
$\eta$ & 7 & - & - \\
$A_{0}$ & 10 & - & - \\
$\lambda_{g}$ & 1 & - & - \\
$r_{\text {min }}$ & $3.50 \cdot 10^{-6}$ & $\mathrm{~m}$ & -
\end{tabular}

Table 2. Global quantities (mean \pm std)

\begin{tabular}{l|c|c} 
total & $\mathcal{M}_{1}$ & $\mathcal{M}_{2}$ \\
\hline length $[\mathrm{m}]$ & $1.94 \pm 0.018$ & $2.68 \pm 0.009$ \\
surface $\left[\mathrm{mm}^{2}\right]$ & $166 \pm 1.00$ & $214 \pm 1.06$ \\
volume $\left[\mathrm{mm}^{3}\right]$ & $1.89 \pm 0.070$ & $2.25 \pm 0.037$ \\
\# terminals & $1.25 \cdot 10^{4} \pm 132$ & $1.84 \cdot 10^{4} \pm 119$
\end{tabular}

Iterative Tree Construction. We use a multiscale approach for the iterative tree construction as summarized in Algorithm 1 . The scaling can be considered as virtual staged growth of the domain (tissue) from a downscaled domain $\Omega_{1}(s=1)$ to the final target domain $\Omega_{N}=\Omega_{0}(s=N)$. For a given arterial tree and scale $s$, the total oxygenation of the simulation domain $\Omega_{s}$ is computed as the superimposition of the oxygenation maps of each individual tree segment according to Equation (3) assuming radial $\mathrm{O}_{2}$ diffusion with a Dirichlet boundary condition on the surface of the artery wall. The $\mathrm{O}_{2}$ concentration inside the vessel lumen is in fact considered constant $c_{1}^{l}=2.04 \cdot 10^{-4} \mathrm{~m}_{\mathrm{O}_{2}}^{3} \mathrm{~m}_{\text {blood }}^{-3}$, assuming $100 \% \mathrm{O}_{2}$ saturation of hemoglobin at a concentration of $1.50 \cdot 10^{4} \mathrm{~g}_{\mathrm{Hb}} \mathrm{m}_{\text {blood }}^{-3}$, and $95 \mathrm{mmHg}$ partial pressure of $\mathrm{O}_{2}$ in blood. The $\mathrm{O}_{2}$ concentration on the surface of the artery wall has been chosen as $25 \% c_{1}^{l}$ which corresponds to a maximum $\mathrm{O}_{2}$ diffusion distance in tissue of approximately $100 \mu \mathrm{m}$ [12]. The induced VEGF steady-state concentration map is subsequently computed according to Equation (5) and used as an excitatory potential field. The tree nodes are sampled "in rounds" with random order within each round to find excited nodes. A node is considered excited if the local VEGF level exceeds a threshold $\theta_{1}$. After the tree has grown at an excited node, the $O_{2}$ and VEGF maps are updated accordingly. The simulation proceeds to the next scale if the $\mathrm{O}_{2}$ supply rate exceeds a scale-dependent level $\kappa_{s}$.

\section{Simulation Results}

We have tested and analyzed the iterative tree construction approach described in Algorithm 1 for different synthetic data sets. A cylindrical simulation domain $\Omega_{0}$ of height and diameter $4.1 \mathrm{~mm}$ has been discretized by a grid with isotropic spacing $32 \mu \mathrm{m}\left(128^{3}\right.$ voxels). The initial trees $\Psi_{0}$ have been defined by six tiny sprouts $(r=5 \mu \mathrm{m})$ on the surface of the cylinder each marking an entry point of a feeding vessel. We have investigated two different configurations for the prescribed metabolic map $\mathcal{R}_{0}$. Model $\mathcal{M}_{1}$ assumes uniform metabolic activity $\mathcal{R}_{0}=R_{1}^{0}$. The metabolic map of model $\mathcal{M}_{2}$ shows an increased level of $\mathrm{O}_{2}$ consumption at the center of the domain. The maximum level 

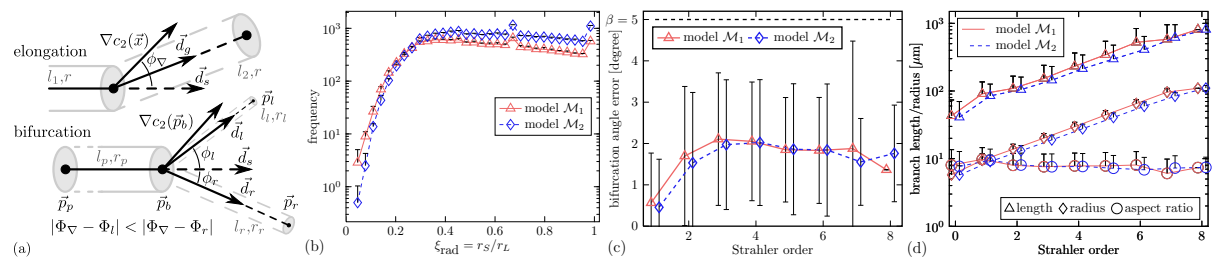

Fig. 1. (a) Apical growth at leaf node. (b) Average frequency of bifurcation symmetry index $\xi_{\text {rad }}$. (c) Average bifurcation angle deviation over diameter-defined Strahler order of the parent segment [5]. The correction threshold $\beta$ is indicated by the dashed line. (d) Average length, radius, and aspect ratio of branches over Strahler order. Upward error bars indicate the standard deviation over all eight simulations per model.

at the center has been set to $11 \cdot R_{1}^{0}$ and decays to $R_{1}^{0}$ according to a Gaussian profile as shown in Figure 3 a. The simulations have been conducted for $N=32$ scales starting at a scaling factor of $1 / 16$ corresponding to an effective voxel spacing of $2 \mu \mathrm{m}$. The supply rate has been computed as the ratio of perfused voxels and the total number of voxels in the domain. A voxel was considered perfused if the effective $O_{2}$ concentration exceeded $t_{1}^{\text {high }}$, i.e., there was no extra VEGF secreted. The scale-dependent target supply rate $\kappa_{s}$ was linearly interpolated between $50 \%(s=1)$ and $95 \%(s=N)$. Defective bifurcation configurations have been readjusted by tree remodeling if the maximum deviation of the left and right bifurcation angle from the optimal bifurcation angle exceeded $\beta=5^{\circ}$.

We have simulated eight realizations of each model $\mathcal{M}_{1}$ and $\mathcal{M}_{2}$ using different seeds for the employed pseudo random number generator (PRNG). The simulation parameters are summarized in Table 1 . Figure 2 visualizes the evolving vasculature at different scales for a single realization of $\mathcal{M}_{2}$ as well as the final tree model of $\mathcal{M}_{1}$.

Despite remarkable variations of the visual appearance of the constructed tree models depending on the PRNG seeds, global geometric quantities such as total lumen volume, surface, and total segment length remained surprisingly stable with virtually no variation for different seeds as summarized in Table 2 However, there are significant differences between the simulation models. The trees constructed for $\mathcal{M}_{2}$ show increased figures w.r.t. the considered global geometric properties. This can be explained by the fact that the trees have to deliver much more oxygen to the tissue corresponding to the increased level of metabolic activity.

We have analyzed the bifurcation pattern in terms of the local symmetry index $\xi_{\text {rad }}=r_{S} / r_{L}$, where $r_{S}$ and $r_{L}$ denote the radius of the smaller and larger distal segment, respectively [5]. The estimated distribution of $\xi_{\text {rad }}$ is shown in Figure 1b. Comparing the bifurcation symmetry, we note that the trees of $\mathcal{M}_{1}$ show an increased number of asymmetric bifurcations (conveying vessels for transport of blood across larger distances), whereas the vasculature of $\mathcal{M}_{2}$ is clearly dominated by symmetric bifurcations (mostly resulting from delivering vessels). The "harmonic" peaks at $\xi_{\text {rad }}=4 / 9,2 / 3,1$ result from a constraint on the choice of bifurcation radii (radii clamped to $\left[r_{\min }, 3 / 2 \cdot r_{p}\right]$ ) to avoid excessive enlargement of the proximal segment.

Tree remodeling, which had become necessary to rebalance the constructed tree after sprouting or bifurcating, has been validated w.r.t. the optimality of bifurcation angles. To this end, the average deviation of the observed bifurcation angles from the optimal 


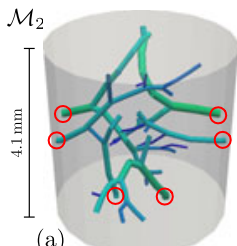

(a)

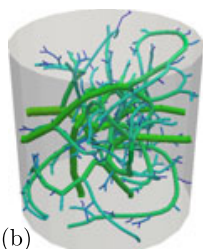

(b)

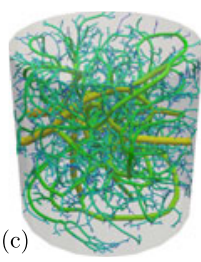

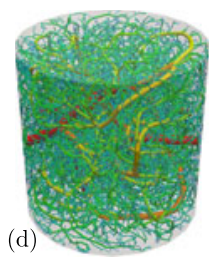

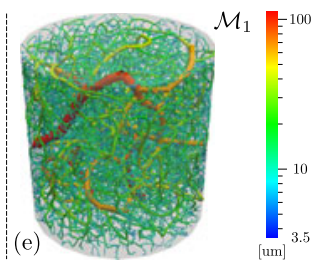

Fig. 2. Evolution of constructed trees for model $\mathcal{M}_{2}$ at scales $s=8,16,24,32$ (a-d). (e) Arterial tree model for model $\mathcal{M}_{1}$ at scale $s=32$. The downscaled simulation domains have been normalized to the size of the target domain $(\mathrm{d}, \mathrm{e})$ for better comparability. The segment radii are color-coded on a logarithmic scale. The entry points of the feeding vessels are marked red (a).

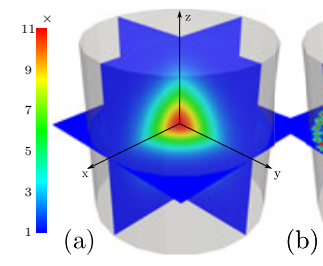

(b)

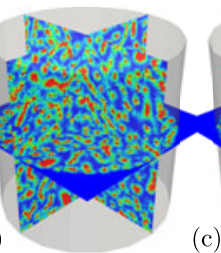

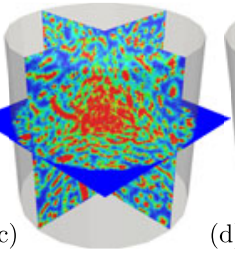

(d)

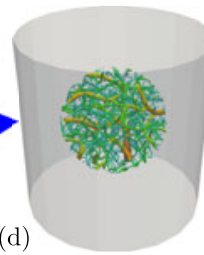

(e)

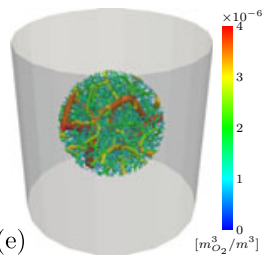

Fig. 3. (a) Synthetic metabolic activity used for model $\mathcal{M}_{2}$. (b,c) Simulated perfusion maps for arterial trees of model $\mathcal{M}_{1}$ (b) and $\mathcal{M}_{2}$ (c). (d,e) Arterial tree models within a centered sphere of radius $1.2 \mathrm{~mm}$ for $\mathcal{M}_{1}$ (d) and $\mathcal{M}_{2}$ (e). The segment radii are color-coded as in Figure 2

configuration has been computed for each bifurcation as shown in Figure 1k. The average angular variations stay well below the correction threshold $\beta=5^{\circ}$ that has been used in the simulations.

It is also interesting to analyze the trees w.r.t. the bifurcation length statistics that has been used for the construction to enforce a certain morphological structure. To this end, we have classified the branches by Strahler order and computed the average length and radius for all classes as reported in Figure $1 \mathrm{~d}$. Length and radius show an exponential profile for both models. The average aspect ratio is almost constant over all Strahler orders at a level of about 10 which corresponds to the normalization factor $A_{0}$ that has been used for our simulations and hence matches the "expected" value $\left(p_{b}\left(A_{0}\right)=0.5\right)$.

The perfusion maps of the artificial tree models are visualized in Figure 3 As opposed to $\mathcal{M}_{1}$ with uniform metabolic activity, the perfusion map for $\mathcal{M}_{2}$ increases towards the center of the domain to meet the prescribed non-uniform metabolic demand. As already indicated by the bifurcation symmetry analysis, the vascular density in the center of the domain is significantly higher for $\mathcal{M}_{2}$ as opposed to $\mathcal{M}_{1}$ (see Figure 3-e).

Explicit comparison of the tree models with real vascular systems is still an open problem. Considering the structural complexity and the inherently stochastic nature of vessel formation, particularly at the capillary level, comparing functional properties and structural statistics might be the best approach to define meaningful similarity measures.

\section{Conclusions and Future Work}

We presented an iterative multi-scale approach for the construction of optimized 3-D arterial tree models based on physiological principles. The driving force of our 
simulations is an angiogenesis model using individual, case-specific information about the metabolic activity of the tissue in the considered domain. Besides, bifurcation statistics and morphological optimality principles are incorporated into the construction. We presented promising results for different synthetic datasets, demonstrating the ability of the algorithm to respect prescribed morphological constraints.

As for future work, there are still many unconsidered aspects, e.g., transition from vascular trees to network-like structures including anastomoses. Likewise, it would be interesting to analyze the fluid dynamic properties of the generated tree models in more detail. The most significant challenge we face is the comparison of the results with experimentally observed real vascular systems w.r.t. structural and functional properties.

Acknowledgements. This work has been funded by the Swiss National Centre of Competence in Research on Computer Aided and Image Guided Medical Interventions (NCCR Co-Me) funded by the Swiss National Science Foundation.

\section{References}

1. Bui, A.V., Manasseh, R., Liffman, K., Sutalo, I.D.: Development of optimized vascular fractal tree models using level set distance function. Med. Eng. Phys. 32(7), 790-794 (2010)

2. Fung, Y.C.: Biomechanics, 2nd edn. Springer, New York (1997)

3. Gabhann, F.M., Ji, J.W., Popel, A.S.: VEGF gradients, receptor activation, and sprout guidance in resting and exercising skeletal muscle. J. Appl. Physiol. 102(2), 722-734 (2007)

4. Ji, J.W., et al.: A computational model of oxygen transport in skeletal muscle for sprouting and splitting modes of angiogenesis. J. Theor. Biol. 241(1), 94-108 (2006)

5. Karch, R., Neumann, F., Neumann, M., Schreiner, W.: Staged growth of optimized arterial model trees. Ann. Biomed. Eng. 28(5), 495-511 (2000)

6. Klarbring, A., Petersson, J., Torstenfelt, B., Karlsson, M.: Topology optimization of flow networks. Comput. Method Appl. M 192(35-36), 3909-3932 (2003)

7. Milde, F., Bergdorf, M., Koumoutsakos, P.: A hybrid model for three-dimensional simulations of sprouting angiogenesis. Biophys. J. 95(7), 3146-3160 (2008)

8. Murray, C.D.: The physiological principle of minimum work: I. The vascular system and the cost of blood volume. Proc. Natl. Acad. Sci. USA 12(3), 207-214 (1926)

9. Nekka, F., Kyriacos, S., Kerrigan, C., Cartilier, L.: A model of growing vascular structures. Bull. Math. Biol. 58(3), 409-424 (1996)

10. Reichold, J., et al.: Vascular graph model to simulate the cerebral blood flow in realistic vascular networks. J. Cereb. Blood Flow Metab. 29(8), 1429-1443 (2009)

11. Schreiner, W., Buxbaum, P.F.: Computer-optimization of vascular trees. IEEE Trans. Biomed. Eng. 40(5), 482-491 (1993)

12. Secomb, T.W., et al.: Green's function methods for analysis of oxygen delivery to tissue by microvascular networks. Ann. Biomed. Eng. 32(11), 1519-1529 (2004)

13. Szczerba, D., Székely, G.: Simulating vascular systems in arbitrary anatomies. In: Duncan, J.S., Gerig, G. (eds.) MICCAI 2005. LNCS, vol. 3750, pp. 641-648. Springer, Heidelberg (2005)

14. Tuchschmid, S., Bajka, M., Szczerba, D., Lloyd, B.A., Székely, G., Harders, M.: Modelling intravasation of liquid distension media in surgical simulators. In: Ayache, N., Ourselin, S., Maeder, A. (eds.) MICCAI 2007, Part I. LNCS, vol. 4791, pp. 717-724. Springer, Heidelberg (2007)

15. Zamir, M.: Optimality principles in arterial branching. J. Theor. Biol. 62(1), 227-251 (1976) 